\title{
Research on Execution and Business Management Theory
}

\author{
Jie Wu
}

Zhengzhou University of Industrial Technology, Zhengzhou, Henan, 451100

\begin{abstract}
Keywords: Execution Ability, Business Management, Enterprise Management, Control Charts, Process Capability
\end{abstract}

\begin{abstract}
Execution is a rich connotation of the concept of fresh and appellation. The idea of implementation has long existed, but in recent years it has attracted the attention of many entrepreneurs and scholars and has gradually become a hot spot of research. This paper explores the issue of the execution of enterprises extensively and strives to accurately grasp the implementation of the connotation and denotation on the basis of in-depth study of the enterprise's executive power of the mechanism, the influencing factors, and how to improve the implementation of the enterprise.
\end{abstract}

\section{Introduction}

In the increasingly fierce market competition today, many business operators found that the company emerged some problems: Why many grand blueprint for the final bubble? Why do many advanced ideas and methodological policies become formalistic when implemented? Why is the commitment of many managers' tasks crushed in the process? Why the same business strategy, the effect has been such a huge difference? Of course, the causes of these problems may be varied, but it is certain that the level of executive power is becoming one of the key factors that enable enterprises to gain a foothold in the highly competitive market. In fact, in most cases, the difference between a company and its competitors rests with the ability of both parties to implement. If your competitor is doing better than you in execution, it will be ahead of you in all aspects. This view is also the unanimous conclusion reached by many entrepreneurs after years of painstaking consideration of the problems that have arisen in their enterprise. Indeed, the implementation of the so-called common concept has left many small and medium-sized enterprises unexpectedly premature when they just got into the fast-moving fast lane. As a result, many state-owned enterprises like air carriers have sunk in the market. This conclusion is the insightful insights exchanged by generations of entrepreneurs with traumatic lessons.

\section{Systematic Analysis of Business Execution}

Study a physical system, we must first study the modalities of the system, that is, to study and determine the internal components of the system, the constitutive relationship between the system and the environment, the internal relations between the variables and their forms of interpretation. An enterprise, just like a physical system, has an open and dynamic complex system as a whole functioning mechanism of internal organization and staffing. No matter how the enterprise's production departments and offices and enterprises and personnel configuration, once the system's static management structure and structural parameters are determined, the dynamic mode of operation of the entire system will be basically determined. Therefore, for a modern enterprise, whether it is a new enterprise or an old enterprise, it is imperative to treat, analyze and grasp it as a real system from a systems engineering point of view. Under certain objective and actual conditions, the classical theory of system control can be completely introduced into the system engineering, and the actual enterprises can be analyzed and applied. This is of great realistic and far-reaching significance to the establishment and improvement of the management mode of modern-day enterprises, the modern management and control of the enterprise system, and the survival and development of the enterprise in the future. In the long run, the same is true for a business and for a country. 
As with computer systems, businesses also have hardware and software systems. The hardware system includes the organizational structure, payroll and rewards and punishment system as well as the design and flow of financial statements. In addition, the communication system is also a part of the hardware. In addition, it also includes the hierarchical distribution of power, such as transparency of authority for task assignment and performance evaluation, rules and regulations. Like a computer system, a company's hardware system must have software to function and become a workable system. Companies in the organizational structure will be divided into different units to carry out different work. However, the design of the structure is important, but it depends on the software to integrate the company into a united and consistent whole. The combination of hardware and software creates social relations, codes of conduct, power relations, and information flows.

Before we discuss the interactive structure of implementation, we must have a profound understanding of the human nature of implementation. Humanity, the essence of man, is an ancient and thought-provoking proposition. Humanity refers to people's distinctions, which are unique to the thing and common to all kinds of things. It includes the natural attributes, that is, the existence, movement, occurrence, development, essence and law of existence in the natural objective material world. Social attributes, such as the life, association, coordination, communication, survival and progress of the social historical world constructed by human beings; Spiritual attributes, that is, the energy, behavior, stimulation, intent, stagnation and so on contained in the human mind. Hypothesis of human nature is based on a certain value orientation, with a certain way of purpose for this kind of prescriptive performance of the choice of abstract reflection. We know that management is an organized social practice carried out by humankind for a certain purpose. People set the subject and object of management activities in one, any proposed management theory, are inseparable from the human nature of knowledge.

\section{Level of Enterprise Executive Power Analysis}

According to different levels of analysis, organizational behavior can be divided into micro-organizational behavior, including individual and group behavior and macro-organizational behavior, the overall behavior of the organization. Accordingly, execution can be divided into three levels: individual execution, departmental (team) execution, and overall business execution. In the introduction, we have analyzed that the execution characteristics of different levels of employees are different. For example, the executive power of the top management focuses on making timely decisions based on the changes in the environment in which the company operates. Their decision-making relates to the rise and fall of the enterprise, with integrity, thus corresponding to the overall execution of the enterprise; middle-level staff more is the decision-making on the basis of accurate understanding of the decomposition, the formation of specific units of the task, Formally manifested in the performance of the department or team; the implementation of enterprise grassroots staff is down to do their own part-time work, it belongs to the scope of the individual implementation. Although personnel at different levels have different characteristics in terms of execution, they all share a commonality in terms of decision-making and implementation: they are both policy-makers and implementers, and have two posts. This is true for any one person in the business. Needless to say, upper-level managers need to make regular decisions for the long-term development of their enterprises. They are decision-makers. In order to put the decision-making into practice, they have to take control measures to check their implementation. At this time, their identities become Executor. The middle of the more so, received the strategic task assigned by the upper, he must make a decision: how to organize resources, the specific implementation to achieve the goal? At this moment, they are the decision-makers; and the process of delegating the grass-roots staff after the plan is established is the implementation process, at which time they are also the executors. For grass-roots personnel, although they only need to do a very specific thing, it seems that they only have the identity of the executor. But then a small thing to determine: what time to do? How to do? Need someone else's cooperation? Identifying these seemingly trivial issues is a decision-making process in its own right, so the bottom-line staff also has the dual role of policymaker and executive. In other words, from business leaders to grassroots employees must 
have a sense of decision-making and implementation, must have the ability to make decisions and implementation.

The upper DEU of the enterprise refers to the top management of the enterprise. Many people have such a misunderstanding: that senior managers as long as they can depict the company's vision, set a good alliance strategy, good management team, and then do a good job of investor relations, the rest of the details of the full implementation of authorization is like, those who do not have to worry about real high Business details of the business. In practice, however, only the top management is fully devoted to the entire production and operation process of the enterprise to ensure the successful implementation of the strategy. Executive top DEU executive skills than the general implementation of middle-level skills and general staff executive skills is more important, many people take it for granted that the enterprise is not strong execution is not subordinate to go according to their superiors, in fact, this is a misunderstanding. Simply throw the task to the staff, of course, will not be effectively implemented. If the management of a task to complete the standard, time is clear, in the process of subordinate to carry out inspection and assistance, and subordinates still can not finish the task, can only say that the task did not account for the real ability to complete this One of the things people should say is that he should find a more suitable person to do, so one of the most important aspects of the execution effect is whether our managers have plans (time planning and standardization), organized (finding the right people Live), leadership (assistance, motivation), and control.

\section{Enterprise's Overall Execution Management}

From the above studies, we can see that the factors influencing the execution of the enterprise are many. Not only the traditional content of the enterprise management will have an impact on the execution ability, but even a contingent event may cause the execution of the enterprise to be harmed. Moreover, the improvement of the execution of enterprises is not an overnight success. It must be achieved through the concerted efforts of all employees of the enterprise, provided that they are adequately prepared for complicated basic work. Therefore, in order to improve the execution ability of enterprises, we must attach importance to this matter from the ideological point of view, treat the execution problems of enterprises as the same as those of other enterprises, and attach importance to the execution management of enterprises as much as other enterprises. At the same time, improving the execution of an enterprise can be combined with other tasks. For example, the implementation of enterprise business processes to take into account the issue of execution, or from the perspective of improving the ability of enterprises to change the organizational structure of enterprises, or the implementation of the evaluation into the individual staff performance appraisal within the scope of. In short, it is necessary to establish an internal implementation of the concept of everyone, there is always the implementation of the driving force of the atmosphere, in order to truly establish an effective implementation of the internal assurance system.

In the reality of management, people are often unconsciously guided by short-term interests while ignoring their long-term interests. This is not only a developing Chinese enterprise, but also a common problem existing in developed country enterprises. Enterprise executive power is a short-term and long-term contradictions. In fact, execution is an effective management of long-term accumulation, rather than short-term emergency behavior and methods to completely solve the problem of business execution, the root causes of long-term corporate culture management must seek answers.

In the management of daily affairs, executive ability can play a very good role in promoting, but a constantly changing competitive environment needs to organize new and ever-changing initiatives. In many cases, it is not able to rapidly adapt to changes in the environment by relying on the concept of change. It is necessary to combine structural changes with cultural changes and create radical changes in enterprises. This radical change of the nature of the enterprise, the demand for the execution of today's enterprises, is not the former managers - the pursuit of a stable meritocratic, but today's leader - to create the destruction of builders, therefore, as business management in order to improve the execution ability of enterprises, the managers in the past must be transformed into 
today's leaders.

At present, many enterprises, regardless of the actual situation, are overly chastened by the trendy and trendy application of management methods. Many business managers are often caught in a misunderstanding, is very fascinated by the management and management methods, and want to find a secret, a shortcut. In fact, there is no management of any secret, just some basic rules and experience of previous experience. As everyone knows, simple and effective, a well-known Japanese entrepreneur once said that if a company encounters bottlenecks and hard-to-solve problems on one hand and finds no suitable solutions for the other, it must learn to return to its origin at the basic point On the way to think and solve and breakthrough, and finally the real way to solve business problems are both simple and obvious. Just like pulling a bow, if you do not pull once, do not force yourself to revert to the status quo; then you can sum up your experience and pull it on again. In fact, management is as simple as that.

\section{Conclusions}

The implementation of any business, whether multinational or domestic enterprises, whether large or small and medium enterprises, the major problems facing the current. Execution does not simply mean simply fulfilling a mission, executing instructions, it is a set of concrete behaviors, techniques and arts. Execution is not a simple tactic, but a set of systematic processes that achieve the goal by asking questions, analyzing problems, and taking actions. Implementation is part of the strategy and the basis of the strategy. Implementation is an important part of linking the strategic goals with the implementation of the strategy. How to implement the corporate strategy, how to turn the company's business objectives into real and visible corporate performance, are closely related to the strength of execution.

\section{References}

[1] Li Jinjun. Application of Balanced Scorecard in Enterprise Performance Management [J]. Journal of Southwest Jiaotong University (Social Sciences)

[2] Wang Zheng, Qi Chunhua. Research on Organizational Decision Support System in Multi-Agent Environment [J]. Machine Tool \& Hydraulics. 2005 (06)

[3] Wang Bo, Jia Xueqin. An effective way to improve the execution ability of enterprise organization [J]. Journal of Chongqing University (Social Science Edition). 2005 (02)

[4] Zhou Shenghui. Self-discipline in business management hypothesis [J]. Business Economics. 2005 (03)

[5] Xiong Suhong, Ma Jun. On the new development of organizational behavior theory [J]. Xinjiang Social Science Forum. 2005 (01) 